\title{
DISTRIBUTED CONTROL OF A HEAT CONDUCTING, TIME-DEPENDENT, NAVIER-STOKES FLUID
}

\author{
RUXANDRA STAVRE \\ Institute of Mathematics, Romanian Academy, P.O. Box 1-764, RO-70700 Bucharest, Romania \\ e-mail: rstavre@stoilow.imar.ro
}

(Received 3 May, 2000; accepted 7 June 2001)

\begin{abstract}
The purpose of this paper is to study an optimal control problem associated with the thermally coupled Navier-Stokes equations. An existence result for this problem is obtained. The most important result of this paper is the proof of the existence and regularity of a solution of the adjoint system. By defining several functions, this system (which is not a divergence free one) is replaced by a divergence free system.
\end{abstract}

2000 Mathematics Subject Classification. 49J20, 49K20, 76D05, 80A20.

1. Introduction. Many papers dealing with optimality conditions for control problems associated with the thermally coupled Navier-Stokes equations have been written in recent years. The problem of minimizing a functional involving the turbulence within the flow was studied in [1] for the stationary case, in [2] for the nonstationary one, the control being the heat flux through the boundary and in [3] for an optimal control in coefficients (the viscosity and the thermal conductivity coefficients). In other papers, such as [7], [4], [5], the cost functional involves the temperature. Why, then, another control problem for the heat-conducting NavierStokes fluid? For at least two reasons. The first one is physical: a lot of practical problems are concerned not with the velocity or the temperature, but with the pressure of the fluid. All the above papers deal with a variational formulation obtained by using the techniques of [8]. In such a variational formulation the unknown pressure does not appear any more.

Let us first describe the physical problem which justifies the study of the control problem considered in the sequel. We have a viscous, incompressible, time-dependent fluid, occupying a bounded, smooth domain $\Omega \subset \mathbb{R}^{2}$. Because of the internal heat sources $g$, the fluid modifies its temperature; hence it modifies also its pressure. We have to answer to the following question: which are the internal heat sources (that cannot be measured) which give a field of the pressure $p$ as close as possible to a desired pressure $p_{d}$ ?

The second reason is of mathematical nature: the obtained adjoint system is an unusual one. The data of this system are not only the data of the problem, but also a function which is not known directly; it can be obtained as a solution of a Neumann problem. Moreover, this system is not a divergence free one, which complicates the proof of the existence and the regularity of a solution for the adjoint problem.

The aim of this paper is to characterize the controls $g$ which give a desired pressure of the fluid. 
The outline of the paper is as follows. In Section 2 we introduce the system describing the problem and the notation. Moreover we give, without proof, some classical theorems concerning this problem. In Section 3 we discuss the proposed control problem and we prove the existence of a solution. Section 4 deals with the necessary conditions of optimality. The most important result of this paper is the proof of the existence and regularity of a solution of the adjoint system. This system is not a divergence free one. To overcome this difficulty, we define several functions which allow us to replace the adjoint system by a divergence free one.

2. The evolution state system. Let $\Omega \subset \mathbb{R}^{2}$ be an open, bounded, connected set with its boundary, $\partial \Omega$ of class $C^{2}$ and $T$ a positive constant. Our physical problem is described by the following coupled system:

$$
\begin{aligned}
& \vec{v}^{\prime}-v \Delta \vec{v}+(\vec{v} \cdot \nabla) \vec{v}+\nabla p=\vec{f}+\vec{B} \tau \text { in } \Omega_{T}=\Omega \times(0, T), \\
& \operatorname{div} \vec{v}=0 \text { in } \Omega_{T}, \\
& \tau^{\prime}-\kappa \Delta \tau+\vec{v} \cdot \nabla \tau=g \text { in } \Omega_{T}, \\
& \vec{v}=\overrightarrow{0}, \quad \tau=0 \text { on } \partial \Omega \times(0, T), \\
& \vec{v}(0)=\overrightarrow{0}, \quad \tau(0)=0 \text { in } \Omega,
\end{aligned}
$$

where $v, \kappa$ are positive constants representing the viscosity of the fluid and the thermal conductivity coefficient, respectively; $\vec{f} \in\left(L^{2}\left(\Omega_{T}\right)\right)^{2}$ represents the body forces, $\vec{B} \in\left(L^{\infty}\left(\Omega_{T}\right)\right)^{2}$ is a function given by the Boussinesq approximation, $g \in L^{2}\left(\Omega_{T}\right)$ represents the internal heat sources and $\vec{v}, \tau, p$ are the unknown of the system (2.1)(2.5), the velocity, the temperature and the pressure of the fluid, respectively.

We shall need in the sequel the following spaces (for their properties see e.g. [8]):

$$
\begin{gathered}
L_{0}^{2}(\Omega)=\left\{u \in L^{2}(\Omega) \mid \int_{\Omega} u \mathrm{~d} x=0\right\}, \\
V=\left\{\vec{u} \in\left(H_{0}^{1}(\Omega)\right)^{2} \mid \operatorname{div} \vec{u}=0\right\}, \\
H=\left\{\vec{u} \in\left(L^{2}(\Omega)\right)^{2}|\operatorname{div} \vec{u}=0, \vec{u} \cdot \vec{n}|_{\partial \Omega}=0\right\}, \\
H^{2,1}\left(\Omega_{T}\right)=\left\{u \in L^{2}\left(\Omega_{T}\right) \mid \frac{\partial u}{\partial t}, \frac{\partial u}{\partial x_{i}}, \frac{\partial^{2} u}{\partial x_{i} \partial x_{j}} \in L^{2}\left(\Omega_{T}\right) ; i, j=1,2\right\},
\end{gathered}
$$

$W\left(0, T ; X, X^{\prime}\right)=\left\{u \in L^{2}(0, T ; X) \mid u^{\prime} \in L^{2}\left(0, T ; X^{\prime}\right)\right\}$ with $X$ a Hilbert space.

The following notation will be used throughout the paper:

$$
\begin{gathered}
(\cdot, \cdot) \text { the scalar product in } L^{2}(\Omega) \text { or }\left(L^{2}(\Omega)\right)^{2}, \\
|\cdot| \text { the norm in } L^{2}(\Omega) \text { or }\left(L^{2}(\Omega)\right)^{2}, \\
((\cdot, \cdot))_{0} \text { the scalar product in } H_{0}^{1}(\Omega) \text { or }\left(H_{0}^{1}(\Omega)\right)^{2}, \\
\|\cdot\|_{0} \text { the norm in } H_{0}^{1}(\Omega) \text { or }\left(H_{0}^{1}(\Omega)\right)^{2},
\end{gathered}
$$


$\langle\cdot, \cdot\rangle_{X, X^{\prime}}$ the duality pairing between a space $X$ and its dual $X^{\prime}$,

$$
\begin{gathered}
b_{1}(\vec{u}, \vec{v})=(\vec{u} \cdot \nabla) \vec{v} \quad \forall \vec{u}, \vec{v} \in\left(H_{0}^{1}(\Omega)\right)^{2}, \\
b_{2}(\vec{u}, \varphi)=\vec{u} \cdot \nabla \varphi \quad \forall \vec{u} \in\left(H_{0}^{1}(\Omega)\right)^{2}, \forall \varphi \in H_{0}^{1}(\Omega) .
\end{gathered}
$$

REMARK 2.1. The existence and uniqueness of a solution of the system (2.1)(2.5) can be obtained for less regular data $\Omega, \vec{f}, \vec{B}, g$ (see e. g. [8], for Navier-Stokes equations). We have chosen this regularity for obtaining the pressure of the fluid $(p)$ at least in $L^{2}\left(\Omega_{T}\right)$. In the last section, this regularity will be improved, in order to give sense to the expression $p(T)$.

The following two theorems establish the existence, uniqueness and regularity of a solution of (2.1)-(2.5) and some a priori estimates, respectively. We shall give them without proofs, since the techniques we use are similar to those of [8], for NavierStokes equations, or of [2] for the coupled system: Navier-Stokes and heat equations for the three dimensional case.

TheOREM 2.2. The system (2.1)-(2.5) has a unique solution $(\vec{v}, \tau, p)$ with $\vec{v} \in\left(H^{2,1}\left(\Omega_{T}\right)\right)^{2} \cap C([0, T] ; V), \quad \tau \in H^{2,1}\left(\Omega_{T}\right) \cap C\left([0, T] ; H_{0}^{1}(\Omega)\right), \quad p \in L^{2}\left(0, T ; H^{1}\right.$ $\left.(\Omega) \cap L_{0}^{2}(\Omega)\right)$.

REMARK 2.3. The uniqueness of the pressure is obtained only in the space $L^{2}\left(0, T ; H^{1}(\Omega) \cap L_{0}^{2}(\Omega)\right)$; as an element of $L^{2}\left(0, T ; H^{1}(\Omega)\right)$ the pressure is unique up to an additive function depending on $t$.

THEOREM 2.4. The unknowns of the problem, $\vec{v}, \tau, p$ satisfy the following estimate:

$$
\begin{aligned}
& \|\vec{v}\|_{\left(H^{2,1}\left(\Omega_{T}\right)\right)^{2}}+\|\tau\|_{H^{2,1}\left(\Omega_{T}\right)}+\|p\|_{L^{2}\left(0, T ; H^{1}(\Omega) \cap L_{0}^{2}(\Omega)\right)} \leq \\
& c(\Omega)\left(\|\vec{f}\|_{\left(L^{2}\left(\Omega_{T}\right)\right)^{2}}+\|\vec{B}\|_{\left(L^{\infty}\left(\Omega_{T}\right)\right)^{2}}+\|g\|_{L^{2}\left(\Omega_{T}\right)}\right) .
\end{aligned}
$$

REMARK 2.5. All the estimates we shall use in the sequel are consequences of the inequality (2.6).

3. Study of the control problem. As mentioned in Section 1, our purpose is to control the pressure of the fluid, by acting on the internal heat sources, $g$. Let $p_{d} \in L^{2}\left(0, T ; L_{0}^{2}(\Omega)\right)$ be the desired configuration of the pressure. Taking into account the uniqueness result stated in Theorem 2.2, the following cost functional

$$
J: L^{2}\left(\Omega_{T}\right) \mapsto \mathbb{R}, J(g)=\frac{1}{2} \int_{\Omega_{T}}\left(p-p_{d}\right)^{2} d x d t
$$

is well defined ( $p$ represents the third component of the unique solution of (2.1)(2.5) corresponding to $g$ ).

Denoting $B_{L^{2}\left(\Omega_{T}\right)}^{r}=\left\{u \in L^{2}\left(\Omega_{T}\right) \mid\|u\|_{L^{2}\left(\Omega_{T}\right)} \leq r\right\}$, we formulate the optimal control problem in the following way: 


$$
\text { Find } g_{0} \in B_{L^{2}\left(\Omega_{T}\right)}^{r} \text { s.t. } J\left(g_{0}\right)=\min \left\{J(g) / g \in B_{L^{2}\left(\Omega_{T}\right)}^{r}\right\} \text {. }
$$

REMARK 3.1. When the cost functional is not coercive, as it happens in our case, there are two ways of introducing the control problem: to look for a minimum point on a bounded set or to consider the following cost functional:

$$
J(g)=\frac{1}{2} \int_{\Omega_{T}}\left(p-p_{d}\right)^{2} d x d t+\frac{N}{2} \int_{\Omega_{T}}\left(g-g_{d}\right)^{2} d x d t .
$$

From the physical point of view the functional defined by (3.3) is not relevant; hence we have chosen the first possibility, which is physically acceptable.

THEOREM 3.2. There exists at least one solution of the control problem (3.2).

Proof. The idea of the proof is to show that $J$ is weakly lower semicontinuous and to use a Weierstrass theorem.

Let $\left\{g_{n}\right\}_{n}$ be a minimizing sequence of $J$ and $\left(\vec{v}_{n}, \tau_{n}, p_{n}\right)$ the unique solution of (2.1)-(2.5) corresponding to $g_{n}$.

Taking into account the compactness of the inclusion $H^{2,1}\left(\Omega_{T}\right) \subset L^{2}\left(\Omega_{T}\right)$ and using the estimates for $\vec{v}_{n}, \tau_{n}$ and $p_{n}$, given by Theorem 2.4, we get the following convergences (on subsequences), as $n \rightarrow \infty$ :

$$
\begin{aligned}
\vec{v}_{n} & \rightarrow \vec{v} \text { weakly in }\left(H^{2,1}\left(\Omega_{T}\right)\right)^{2}, \\
\vec{v}_{n} & \rightarrow \vec{v} \text { strongly in }\left(L^{2}\left(\Omega_{T}\right)\right)^{2}, \\
\tau_{n} & \rightarrow \tau \text { weakly in } H^{2,1}\left(\Omega_{T}\right), \\
p_{n} & \rightarrow p \text { weakly in } L^{2}\left(\Omega_{T}\right),
\end{aligned}
$$

and, of course,

$$
g_{n} \rightarrow g \text { weakly in } L^{2}\left(\Omega_{T}\right) .
$$

In order to use these convergences, we shall write the weak formulation of (2.1)(2.5) in the following form:

$$
\begin{aligned}
& (\vec{v}(t), \vec{z})+v((\vec{v}(t), \vec{z}))_{0}+\left(b_{1}(\vec{v}(t), \vec{v}(t)), \vec{z}\right)-(p(t), \operatorname{div}, \vec{z})= \\
& (\vec{f}(t), \vec{z})+(\vec{B}(t) \tau(t), \vec{z}) \quad \forall \vec{z} \in\left(H_{0}^{1}(\Omega)\right)^{2}, \\
& (\operatorname{div}, \vec{v}(t), s)=0 \quad \forall s \in L^{2}(\Omega) \text {, } \\
& \left(\tau^{\prime}(t), \eta\right)+\kappa((\tau(t), \eta))_{0}+\left(b_{2}(\vec{v}(t), \tau(t)), \eta\right)=(g(t), \eta) \quad \forall \eta \in H_{0}^{1}(\Omega), \\
& \vec{v}(0)=\overrightarrow{0}, \tau(0)=0 \text { in } \Omega \text {. }
\end{aligned}
$$

Using Lemma $3.2[8$, p. 289] we can pass to the limit, as $n \rightarrow \infty$, in (3.4)-(3.7), written for $g=g_{n}$, and we get the weakly lower semicontinuity of the cost functional; thus the assertion of the theorem is obtained.

The last result of this section is the differentiability of the cost functional. 
Proposition 3.3. The functional $J$ is differentiable and

$$
\left(J^{\prime}\left(g_{0}\right), h\right)_{L^{2}\left(\Omega_{T}\right)}=\int_{\Omega_{T}}\left(p_{h}^{*}-p_{0}\right)\left(p_{0}-p_{d}\right), d x d t \quad \forall h, g_{0} \in L^{2}\left(\Omega_{T}\right),
$$

where $p_{0}$ is the third component of the unique solution of (2.1)-(2.5) corresponding to $g_{0}$ and $\left(\vec{v}_{h}^{*}, \tau_{h}^{*}, p_{h}^{*}\right)$ is the unique solution of the following system:

$$
\begin{aligned}
\vec{v}_{h}^{* \prime}-v \triangle \vec{v}_{h}^{*}+b_{1}\left(\vec{v}_{h}^{*}, \vec{v}_{0}\right)+b_{1}\left(\vec{v}_{0}, \vec{v}_{h}^{*}\right)+\nabla p_{h}^{*} & =\vec{B} \tau_{h}^{*}+\vec{f}+b_{1}\left(\vec{v}_{0}, \vec{v}_{0}\right), \text { in } \Omega_{T}, \\
\operatorname{div}, \vec{v}_{h}^{*} & =0 \text { in } \Omega_{T}, \\
\tau_{h}^{* \prime}-\kappa \Delta \tau_{h}^{*}+b_{2}\left(\vec{v}_{h}^{*}, \tau_{0}\right)+b_{2}\left(\vec{v}_{0}, \tau_{h}^{*}\right) & =h+g_{0}+b_{2}\left(\vec{v}_{0}, \tau_{0}\right) \text { in } \Omega_{T}, \\
\vec{v}_{h}^{*}(0) & =\overrightarrow{0}, \tau_{h}^{*}(0)=0 \text { in } \Omega, \\
\vec{v}_{h}^{*} & =\overrightarrow{0}, \tau_{h}^{*}=0 \text { on } \partial \Omega \times(0, T) .
\end{aligned}
$$

Proof. For any $\alpha \in(0,1)$ and $h, g_{0} \in L^{2}\left(\Omega_{T}\right)$ we denote by $\left(\vec{v}_{0}, \tau_{0}, p_{0}\right)$ the unique solution of (2.1)-(2.5) corresponding to $g=g_{0}$ and by $\left(\vec{v}_{\alpha h}, \tau_{\alpha h}, p_{\alpha h}\right)$ the solution for $g=g_{0}+\alpha h$. Let $\vec{v}_{\alpha}, \tau_{\alpha}, p_{\alpha}$ be the following functions:

$$
\vec{v}_{\alpha}=\frac{\vec{v}_{\alpha h}-\vec{v}_{0}}{\alpha}+\vec{v}_{0}, \quad \tau_{\alpha}=\frac{\tau_{\alpha h}-\tau_{0}}{\alpha}+\tau_{0}, \quad p_{\alpha}=\frac{p_{\alpha h}-p_{0}}{\alpha}+p_{0} .
$$

A direct computation gives the system satisfied by $\left(\vec{v}_{\alpha}, \tau_{\alpha}, p_{\alpha}\right)$, which is of the same type as (2.1)-(2.5). Hence we obtain for $\vec{v}_{\alpha}, \tau_{\alpha}, p_{\alpha}$ the properties given by Theorems 2.2. and 2.4., with the constant $c(\Omega)$ in (2.6) not depending on $\alpha$. The boundedness of $\left(\vec{v}_{\alpha}, \tau_{\alpha}, p_{\alpha}\right)$ in $\left(H^{2,1}\left(\Omega_{T}\right)\right)^{2} \times H^{2,1}\left(\Omega_{T}\right) \times L^{2}\left(0, T ; L_{0}^{2}(\Omega)\right)$ yields the existence of $\left(\vec{v}_{h}^{*}, \tau_{h}^{*}, p_{h}^{*}\right)$ in this space such that the following convergences hold, as $\alpha \searrow 0$, on subsequences:

$$
\begin{aligned}
& \vec{v}_{\alpha} \rightarrow \vec{v}_{h}^{*} \text { weakly in }\left(H^{2,1}\left(\Omega_{T}\right)\right)^{2}, \\
& \tau_{\alpha} \rightarrow \tau_{h}^{*} \text { weakly in } H^{2,1}\left(\Omega_{T}\right), \\
& p_{\alpha} \rightarrow p_{h}^{*} \text { weakly in } L^{2}\left(\Omega_{T}\right) .
\end{aligned}
$$

Computing $\lim _{\alpha \searrow 0} \frac{J\left(g_{0}+\alpha h\right)-J\left(g_{0}\right)}{\alpha}$ and passing to the limit, as $\alpha \searrow 0$, in the weak formulation of the system with the solution $\left(\vec{v}_{\alpha}, \tau_{\alpha}, p_{\alpha}\right)$, we achieve the conclusion of the proposition.

Let $g_{0}$ be an optimal control. Denoting by $\left(\vec{v}^{*}, \tau^{*}, p^{*}\right)$ the solution of (3.9)-(3.13) corresponding to $h=g-g_{0}$ and using (3.8), we obtain the following inequality:

$$
\int_{\Omega_{T}}\left(p^{*}-p_{0}\right)\left(p_{0}-p_{d}\right), d x d t \geq 0
$$

In the last section, this inequality will be replaced by an inequality without constraints, by introducing the adjoint system. 
4. First-order necessary conditions of optimal solutions. Let $g_{0}$ be an optimal control and $\left(\vec{v}_{0}, \tau_{0}, p_{0}\right)$ the unique solution of (2.1)-(2.5) corresponding to $g_{0}$. As mentioned in Remark 2.1, in this section we shall need further regularity for the data: $\vec{f}, \vec{B}, p_{d}$.

Proposition 4.1. For $\vec{f}, \vec{f}^{\prime} \in\left(L^{2}\left(\Omega_{T}\right)\right)^{2}, \vec{f}(0) \in V, \vec{B}, \vec{B}^{\prime} \in\left(L^{\infty}\left(\Omega_{T}\right)\right)^{2}$ it follows $p_{0}^{\prime} \in L^{2}\left(\Omega_{T}\right)$.

Proof. For $\vec{f}$ and $\vec{B}$ with the above regularity and taking into account the regularity of $\tau_{0}$ given by Theorem 2.2., we can apply Theorem $3.5[8$, p. 299] and we get $\vec{v}_{0}^{\prime} \in L^{2}(0, T ; V) \cap L^{\infty}(0, T ; H)$.

We consider now the derivative with respect to the time variable of equation (2.1). Denoting by $\vec{U}=\vec{v}_{0}^{\prime}, P=p_{0}^{\prime}, \vec{F}=\vec{f}^{\prime}+\vec{B}^{\prime} \tau_{0}+\vec{B} \tau_{0}^{\prime}$, we obtain in a distributional sense the following equation:

$$
\vec{U}^{\prime}-v \Delta \vec{U}+b_{1}\left(\vec{U}, \vec{v}_{0}\right)+b_{1}\left(\vec{v}_{0}, \vec{U}\right)-\nabla P=\vec{F},
$$

with $\vec{U} \in L^{2}(0, T ; V) \cap L^{\infty}(0, T ; H)$.

The regularity of $\vec{v}_{0}$ together with the above regularity of $\vec{U}$ give, as in Lemma $3.4\left[8\right.$, p. 292], $b_{1}\left(\vec{v}_{0}, \vec{U}\right), b_{1}\left(\vec{U}, \vec{v}_{0}\right) \in L^{2}\left(0, T ; V^{\prime}\right)$.

Since $\vec{F} \in\left(L^{2}\left(\Omega_{T}\right)\right)^{2}$, with the same techniques as in Theorem 3.2 [8, p. 294] we obtain $\vec{U}^{\prime} \in L^{2}\left(0, T ; V^{\prime}\right)$ and hence $\vec{U}(0) \in H$.

Finally, from $\vec{f}(0) \in V$ it follows $\vec{U}(0) \in V$ and, as in Theorem 2.2, we obtain at least the regularity $P \in L^{2}\left(\Omega_{T}\right)$, which completes the proof.

It can be easily proved that, if $p_{0} \in L^{2}\left(0, T ; L_{0}^{2}(\Omega)\right)$ and $p_{0}^{\prime} \in L^{2}\left(\Omega_{T}\right)$, then $p_{0}^{\prime} \in L^{2}\left(0, T ; L_{0}^{2}(\Omega)\right)$.

Let $p_{d}^{\prime}$ be an element of $L^{2}\left(\Omega_{T}\right)$; hence $p_{0}(T)-p_{d}(T)$ makes sense as an element of $L_{0}^{2}(\Omega)$.

We introduce the following adjoint system:

$$
\begin{aligned}
& \vec{w} \in L^{2}\left(0, T ;\left(H_{0}^{1}(\Omega)\right)^{2}\right), \varphi \in L^{2}\left(0, T ; H_{0}^{1}(\Omega)\right), \pi \in \mathcal{D}^{\prime}\left(\Omega_{\mathcal{T}}\right), \\
& -\vec{w}^{\prime}-v \Delta \vec{w}-b_{1}\left(\vec{v}_{0}, \vec{w}\right)+\left(\nabla \vec{v}_{0}\right)^{T} \vec{w}+\nabla \pi-\tau_{0} \nabla \varphi=0 \text { in } \Omega_{T}, \\
& \operatorname{div} \vec{w}=p_{0}-p_{d} \text { in } \Omega_{T}, \\
& -\varphi^{\prime}-\kappa \Delta \varphi-b_{2}\left(\vec{v}_{0}, \varphi\right)-\vec{B} \cdot \vec{w}=0 \text { in } \Omega_{T}, \\
& \vec{w}(T)=\nabla G, \varphi(T)=0 \text { in } \Omega,
\end{aligned}
$$

where $G$ is the unique solution (up to an additive constant) of the Neumann problem

$$
\begin{aligned}
G & \in H^{1}(\Omega), \\
\triangle G & =p_{0}(T)-p_{d}(T) \text { in } \Omega, \\
\frac{\partial G}{\partial n} & =0 \text { on } \partial \Omega .
\end{aligned}
$$

REMARK 4.2. In order to make sense of the expression $\vec{w}(T)$ as an element of $L^{2}(\Omega)$ it seems to be necessary to have the regularity condition $\vec{w}^{\prime} \in L^{2}\left(0, T ;\left(H^{-1}(\Omega)\right)^{2}\right)$. In fact, we shall obtain only the weaker condition $\vec{w}^{\prime} \in L^{2}\left(0, T ; V^{\prime}\right)$, but this is sufficient since $\vec{w} \in C\left([0, T] ;\left(L^{2}(\Omega)\right)^{2}\right)$. 
Because of the equation (4.3), we cannot give a variational formulation of the system (4.1)-(4.5) with test functions in $V$ and then, obtain existence and regularity results using the Galerkin's approximation.

In the case of stationary flows, the system with $\operatorname{div} \vec{v} \neq 0$ is easily replaced by a divergence free system (see [8, p. 31]). We don't use the same technique in our case, since we need also regularity results for the time derivative, properties which cannot be obtained with this method.

We consider the following problem (a. e. in $(0, T))$ :

$$
\begin{aligned}
\operatorname{div} \vec{z}(t) & =\left(p_{0}(t)-p_{d}(t)\right)^{\prime} \text { in } \Omega, \\
\vec{z}(t) & =\overrightarrow{0} \text { on } \partial \Omega .
\end{aligned}
$$

Taking into account that $\left(p_{0}(t)-p_{d}(t)\right)^{\prime} \in L_{0}^{2}(\Omega)$ and using a classical result (see e.g. [8, p. 32]) we obtain the existence of a unique $\vec{z}_{p}(t) \in\left(H_{0}^{1}(\Omega)\right)^{2}$ such that:

$$
\left\|\vec{z}_{p}(t)\right\|_{0} \leq c(\Omega)\left|\left(p_{0}(t)-p_{d}(t)\right)^{\prime}\right|
$$

Since $\left(p_{0}-p_{d}\right)^{\prime} \in L^{2}\left(\Omega_{T}\right)$ it follows, from (4.8), that $\vec{z}_{p} \in L^{2}\left(0, T ;\left(H_{0}^{1}(\Omega)\right)^{2}\right)$.

We define a new function, given by

$$
\vec{u}_{p}(t)=\int_{T}^{t} \vec{z}_{p}(r) \mathrm{dr}, \text { a. e. in }(0, T)
$$

and we obtain, without difficulty, the regularity $\vec{u}_{p} \in L^{2}\left(0, T ;\left(L^{2}(\Omega)\right)^{2}\right)$. Moreover, by using a standard result (see e.g. $\left[\begin{array}{l}6 \\ \text { p. 566 }\end{array}\right)$, it follows: $\nabla u_{p}^{i}(t)=$ $\int_{T}^{t} \nabla z_{p}^{i}(r) d r, i=1,2$. Taking into account the regularity of $\vec{z}_{p}$, it is easy now to verify that $\vec{u}_{p} \in L^{2}\left(0, T ;\left(H_{0}^{1}(\Omega)\right)^{2}\right)$. On the other hand, the definition (4.9) also gives some regularity for $\vec{u}_{p}^{\prime}$, i.e. $\vec{u}_{p}^{\prime} \in L^{2}\left(0, T ;\left(H_{0}^{1}(\Omega)\right)^{2}\right)$. Hence we defined a function with the following properties: $\vec{u}_{p}(T)=\overrightarrow{0}, \vec{u}_{p}, \vec{u}_{p}^{\prime} \in L^{2}\left(0, T ;\left(H_{0}^{1}(\Omega)\right)^{2}\right)$ and

$$
\left\|\vec{u}_{p}\right\|_{L^{2}\left(0, T ;\left(H_{0}^{1}(\Omega)\right)^{2}\right)}+\left\|\vec{u}_{p}^{\prime}\right\|_{L^{2}\left(0, T ;\left(H_{0}^{1}(\Omega)\right)^{2}\right)} \leq c(\Omega, T)\left\|\left(p_{0}-p_{d}\right)^{\prime}\right\|_{L^{2}\left(\Omega_{T}\right)} .
$$

We introduce another function, $\vec{\psi}_{p}$, as the unique solution of the problem

$$
\begin{array}{r}
\operatorname{div} \vec{\psi}=p_{0}(T)-p_{d}(T) \text { in } \Omega, \\
\vec{\psi}=\overrightarrow{0} \text { on } \partial \Omega,
\end{array}
$$

satisfying the estimate

$$
\|\vec{\psi}\|_{0} \leq c(\Omega)\left|p_{0}(T)-p_{d}(T)\right|
$$

Computing $\operatorname{div} \vec{u}_{p}(t)$, we get, from (4.9) the following equality: $\operatorname{div} \vec{u}_{p}(t)=$ $\left(p_{0}(t)-p_{d}(t)\right)-\left(p_{0}(T)-p_{d}(T)\right)$ almost everywhere in $(0, T)$; hence, using (4.11) we obtain:

$$
\operatorname{div}\left(\vec{u}_{p}(t)+\vec{\psi}_{p}\right)=p_{0}(t)-p_{d}(t) \text { a. e. in }(0, T)
$$


Let us define a new function:

$$
\vec{v}_{p}(t)=\vec{u}_{p}(t)+\vec{\psi}_{p} \text { a. e. in }(0, T) .
$$

Taking into account the properties of $\vec{u}_{p}$ and $\vec{\psi}_{p}$ we obtain: $\vec{v}_{p}, \vec{v}_{p}^{\prime} \in L^{2}(0, T$; $\left.\left(H_{0}^{1}(\Omega)\right)^{2}\right) ; \operatorname{div} \vec{v}_{p}(t)=p_{0}(t)-p_{d}(t)$ a. e. in $(0, T) ; \vec{v}_{p}(T)=\vec{\psi}_{p}$ in $\left(L^{2}(\Omega)\right)^{2}$.

We are now in a position to introduce the following divergence free system:

$$
\begin{aligned}
& \vec{y} \in W\left(0, T ; V, V^{\prime}\right), \varphi \in W\left(0, T ; H_{0}^{1}(\Omega), H^{-1}(\Omega)\right), \pi \in \mathcal{D}^{\prime}\left(\Omega_{\mathcal{T}}\right), \\
& -\vec{y}^{\prime}-v \Delta \vec{y}-b_{1}\left(\vec{v}_{0}, \vec{y}\right)+\left(\nabla \vec{v}_{0}\right)^{T} \vec{y}+\nabla \pi-\tau_{0} \nabla \varphi=\vec{f}_{p} \text { in } \Omega_{T}, \\
& -\varphi^{\prime}-\kappa \Delta \varphi-b_{2}\left(\vec{v}_{0}, \varphi\right)-\vec{B} \cdot \vec{y}=g_{p} \text { in } \Omega_{T}, \\
& \vec{y}(T)=\vec{y}_{T}, \varphi(T)=0 \text { in } \Omega,
\end{aligned}
$$

where

$$
\begin{aligned}
\vec{f}_{p} & =\vec{v}_{p}^{\prime}+v \Delta \vec{v}_{p}+b_{1}\left(\vec{v}_{0}, \vec{v}_{p}\right)-\left(\nabla \vec{v}_{0}\right)^{T} \vec{v}_{p}, \\
g_{p} & =\vec{B} \cdot \vec{v}_{p}, \\
\vec{y}_{T} & =\nabla G-\vec{\psi}_{p},
\end{aligned}
$$

with $\vec{v}_{p}, \vec{\psi}_{p}$ and $G$ previously defined.

Using the regularity of $\vec{v}_{0}, \vec{v}_{p}, \vec{B}$ and some classical results of [8] we obtain at least $\vec{f}_{p} \in L^{2}\left(0, T ; V^{\prime}\right)$ and $g_{p} \in L^{2}\left(\Omega_{T}\right)$. For $\vec{y}_{T}$ we have the regularity $\vec{y}_{T} \in H$. Indeed, $\operatorname{div} \vec{y}_{T}=\triangle G-\operatorname{div} \vec{\psi}_{p}=0$ in $\Omega$ and $\vec{y}_{T} \cdot \vec{n}=\frac{\partial G}{\partial n}-\vec{\psi}_{p} \cdot \vec{n}=0$ on $\partial \Omega$.

Now we can state the main result of this paper:

THEOREM 4.3. The adjoint system (4.1)-(4.5) has at least a solution $(\vec{w}, \varphi, \pi)$, with $\vec{w}^{\prime} \in L^{2}\left(0, T ; V^{\prime}\right), \vec{w} \in C\left([0, T] ;\left(L^{2}(\Omega)\right)^{2}\right) ; \varphi^{\prime} \in L^{2}\left(0, T ; H^{-1}(\Omega)\right)$ and $\vec{w}, \varphi$ unique.

Proof. The regularity of the data $\vec{f}_{p}, g_{p}$ and $\vec{y}_{T}$ and some standard results for Navier-Stokes equations (see [8]) allow us to obtain the existence and uniqueness of the function $\vec{y}$ in $W\left(0, T ; V, V^{\prime}\right)$, the existence and uniqueness of the function $\varphi$ in $W\left(0, T ; H_{0}^{1}(\Omega), H^{-1}(\Omega)\right)$ and the existence of a distribution $\pi$, satisfying the system (4.15)-(4.18).

By putting

$$
\vec{w}=\vec{y}+\vec{v}_{p},
$$

it is easy to verify that $\vec{w} \in C\left([0, T] ;\left(L^{2}(\Omega)\right)^{2}\right)$ and $\vec{w}^{\prime} \in L^{2}\left(0, T ; V^{\prime}\right)$; moreover $(\vec{w}, \varphi, \pi)$ is a solution of the adjoint system (4.1)-(4.5).

The uniqueness of $\vec{w}$ and $\varphi$ is obtained as follows. Let $\left(\vec{w}_{1}, \varphi_{1}, \pi_{1}\right)$ and $\left(\vec{w}_{2}, \varphi_{2}, \pi_{2}\right)$ be two solutions of the system (4.1)-(4.5). We denote $(\vec{w}, \varphi, \pi)=$ $\left(\vec{w}_{1}, \varphi_{1}, \pi_{1}\right)-\left(\vec{w}_{2}, \varphi_{2}, \pi_{2}\right)$ and by subtracting the variational formulations of the adjoint system for $\left(\vec{w}_{1}, \varphi_{1}, \pi_{1}\right)$ and $\left(\vec{w}_{2}, \varphi_{2}, \pi_{2}\right)$ we get:

$$
\begin{aligned}
& \left.-\left\langle\vec{w}^{\prime}(t), \vec{z}\right\rangle_{V, V^{\prime}}+v((\vec{w}(t), \vec{z}))_{0}-b_{1}\left(\vec{v}_{0}(t), \vec{w}(t)\right), \vec{z}\right)+ \\
& \left\langle\left(\nabla \vec{v}_{0}(t)\right)^{T} \vec{w}(t), \vec{z}\right\rangle_{V, V^{\prime}}-\left(\tau_{0}(t) \nabla \varphi(t), \vec{z}\right)=0 \quad \forall \vec{z} \in V,
\end{aligned}
$$




$$
\begin{aligned}
(\operatorname{div}, \vec{w}(t), s) & =0 \quad \forall s \in L^{2}(\Omega), \\
-\left\langle\varphi^{\prime}(t), \eta\right\rangle_{H^{-1}(\Omega), H_{0}^{1}(\Omega)} & \left.+\kappa((\varphi(t), \eta))_{0}-b_{2}\left(\vec{v}_{0}(t), \varphi(t)\right), \eta\right)- \\
(\vec{B}(t) \cdot \vec{w}(t), \eta) & =0 \quad \forall \eta \in H_{0}^{1}(\Omega), \\
\vec{w}(T) & =\overrightarrow{0}, \quad \varphi(T)=0 \text { in } \Omega .
\end{aligned}
$$

The desired result is now obtained taking $\vec{z}=\vec{w}(t), \eta=\varphi(t)$ and using standard techniques.

The last result of this paper states the optimality conditions satisfied by a solution $g_{0}$ of (3.2).

THEOREm 4.4. Let us suppose that $g_{0}$ is an optimal control. Then there exists the unique elements

$$
\begin{gathered}
\left(\vec{v}_{0}, \tau_{0}, p_{0}\right) \in\left(H^{2,1}\left(\Omega_{T}\right)\right)^{2} \cap C([0, T] ; V) \times H^{2,1}\left(\Omega_{T}\right) \times L^{2}\left(0, T ; H^{1}(\Omega) \cap L_{0}^{2}(\Omega)\right) \\
\left(\vec{w}_{0}, \varphi_{0}\right) \in L^{2}\left(0, T ;\left(H_{0}^{1}(\Omega)\right)^{2}\right) \cap C\left([0, T] ;\left(L^{2}(\Omega)\right)^{2}\right) \times W\left(0, T ; H_{0}^{1}(\Omega), H^{-1}(\Omega)\right),
\end{gathered}
$$

with $\vec{w}_{0}^{\prime} \in L^{2}\left(0, T ; V^{\prime}\right)$ and a unique distribution $\pi_{0}$ up to an additive distribution in $(0, T)$ such that the following system is satisfied:

$$
\begin{gathered}
\vec{v}_{0}^{\prime}-v \Delta \vec{v}_{0}+b_{1}\left(\vec{v}_{0}, \vec{v}_{0}\right)+\nabla p_{0}=\vec{f}+\vec{B} \tau_{0} \text { in } \Omega_{T}, \\
\operatorname{div} \vec{v}_{0}=0 \text { in } \Omega_{T}, \\
\tau_{0}^{\prime}-\kappa \Delta \tau_{0}+b_{2}\left(\vec{v}_{0}, \tau_{0}\right)=g_{0} \text { in } \Omega_{T}, \\
\vec{v}_{0}(0)=\overrightarrow{0}, \tau_{0}(0)=0 \text { in } \Omega, \\
-\vec{w}_{0}^{\prime}-v \Delta \vec{w}_{0}-b_{1}\left(\vec{v}_{0}, \vec{w}_{0}\right)+\left(\nabla \vec{v}_{0}\right)^{T} \vec{w}_{0}+\nabla \pi_{0}-\tau_{0} \nabla \varphi_{0}=0 \text { in } \Omega_{T}, \\
\operatorname{div} \vec{w}_{0}=p_{0}-p_{d} \text { in } \Omega_{T}, \\
-\varphi_{0}^{\prime}-\kappa \Delta \varphi_{0}-b_{2}\left(\vec{v}_{0}, \varphi_{0}\right)-\vec{B} \cdot \vec{w}_{0}=0 \text { in } \Omega_{T}, \\
\vec{w}_{0}(T)=\nabla G, \varphi_{0}(T)=0 \text { in } \Omega, \\
\int_{\Omega_{T}} \varphi_{0}\left(g_{0}-g\right) d x d t \geq 0 \forall g \in B_{L^{2}\left(\Omega_{T}\right)}^{r} .
\end{gathered}
$$

Proof. The first assertion of the theorem has already been obtained. We have to prove the inequality (4.29). For this purpose we consider the weak formulations of systems (3.9)-(3.13), (4.27), (4.28). Taking adequate test functions in these variational formulations, after an easy computation, we get

$$
\begin{aligned}
& \left(p_{0}(t)-p_{d}(t), p^{*}(t)-p_{0}(t)\right)=\left(\varphi_{0}(t), g_{0}(t)-g(t)\right)- \\
& \left\langle\varphi_{0}^{\prime}(t), \tau_{0}(t)-\tau^{*}(t)\right\rangle_{H^{-1}(\Omega), H_{0}^{1}(\Omega)}-\left(\left(\tau_{0}(t)-\tau^{*}(t)\right)^{\prime}, \varphi_{0}(t)\right)- \\
& \left\langle\vec{w}_{0}^{\prime}(t), \vec{v}_{0}(t)-\vec{v}^{*}(t)\right\rangle_{V^{\prime}, V}-\left(\left(\vec{v}_{0}(t)-\vec{v}^{*}(t)\right)^{\prime}, \vec{w}_{0}(t)\right) .
\end{aligned}
$$

Integrating the equality (4.30) from 0 to $T$ and using a Green's formula, it follows:

$$
\begin{aligned}
& \int_{0}^{T}\left(p_{0}-p_{d}\right)\left(p^{*}-p_{0}\right) d x d t=\int_{0}^{T} \varphi_{0}\left(g-g_{0}\right) d x d t-\left(\vec{w}_{0}(T), \vec{v}_{0}(T)-\vec{v}^{*}(T)\right)= \\
& \int_{0}^{T} \varphi_{0}\left(g_{0}-g\right) d x d t-\left(\nabla G, \vec{v}_{0}(T)-\vec{v}^{*}(T)\right)=
\end{aligned}
$$




$$
\int_{0}^{T} \varphi_{0}\left(g_{0}-g\right) d x d t+\left(G, \operatorname{div}\left(\vec{v}_{0}(T)-\vec{v}^{*}(T)\right)\right)=\int_{0}^{T} \varphi_{0}\left(g_{0}-g\right) d x d t
$$

since $\vec{v}_{0}-\vec{v}^{*} \in C([0, T] ; V)$ and $\frac{\partial G}{\partial n}=0$ on $\partial \Omega$. Therefore the theorem is proved.

\section{REFERENCES}

1. F. Abergel and E. Casas, Some optimal control problems of multistate equations appearing in fluid mechanics, M2AN 27 (1993), 223-247.

2. E. Casas, The Navier-Stokes equations coupled with the heat equation: analysis and control, Control and Cybernet. 23 (1994), 605-620.

3. A. Căpăţînă and R. Stavre, Optimality conditions for control problems in heat conducting Navier-Stokes fluid, Rev. Roumaine Sci. Tech.-Méc. Appl. 40 (1995), 189-199.

4. A. Căpăţînă and R. Stavre, Optimal control of a non-isothermal Navier-Stokes flow, Int. J. Engrg. Sci. 34 (1996), 59-66.

5. A. Căpăţină and R. Stavre, Numerical analysis of a control problem in heat-conducting Navier-Stokes fluid, Int. J. Engrg. Sci. 34 (1996), 1467-1476.

6. R. Dautray and J. L. Lions, Analyse mathématique et calcul numérique pour les sciences et les techniques (Masson, Paris, Milan, Barcelone, Mexico, 1988).

7. K. Ito, Boundary temperature control for thermally coupled Navier-Stokes equations, Internat. Ser. Num. Math. 118 (1994), 211-230.

8. R. Temam, Navier-Stokes equations (North-Holland, Amsterdam, 1977). 